\section{Cognitive Function Following Diabetic Ketoacidosis in Children With New-Onset or Previously Diagnosed Type 1 Diabetes}

Diabetes Care 2020;43:2768-2775 | https://doi.org/10.2337/dc20-0187
Simona Ghetti, ${ }^{1,2}$ Nathan Kuppermann, ${ }^{3,4}$ Arleta Rewers, ${ }^{5}$ Sage R. Myers, ${ }^{6}$ Jeff E. Schunk, ${ }^{7}$ Michael J. Stoner, ${ }^{8}$ Aris Garro, ${ }^{9}$ Kimberly S. Quayle, ${ }^{10}$ Kathleen M. Brown, ${ }^{11}$ Jennifer L. Trainor, ${ }^{12}$ Leah Tzimenatos, ${ }^{3}$ Andrew D. DePiero, ${ }^{13}$ Julie K. McManemy, ${ }^{14}$ Lise E. Nigrovic, ${ }^{15}$ Maria Y. Kwok, ${ }^{16}$ Clinton S. Perry III, ${ }^{2,17}$ Cody S. Olsen, ${ }^{7}$ T. Charles Casper, ${ }^{7}$ and Nicole S. Glaser, ${ }^{4}$ for the Pediatric Emergency Care Applied Research Network (PECARN) DKA FLUID Study Group*

\section{OBJECTIVE}

This study assessed whether a single diabetic ketoacidosis (DKA) episode is associated with cognitive declines in children with newly diagnosed type 1 diabetes and whether the same is true in children who had previously been diagnosed after accounting for variations in glycemic control and other relevant factors.

\section{RESEARCH DESIGN AND METHODS}

We prospectively enrolled 758 children, 6-18 years old, who presented with DKA in a randomized multisite clinical trial evaluating intravenous fluid protocols for DKA treatment. DKA was moderate/severe in $\mathbf{4 3 0}$ children and mild in 328 children. A total of 392 children with DKA had new onset of type 1 diabetes, and the rest were previously diagnosed. Neurocognitive assessment occurred 2-6 months after the DKA episode. A comparison group of 376 children with type 1 diabetes, but no DKA exposure, was also enrolled.

\section{RESULTS}

Among all patients, moderate/severe DKA was associated with lower intelligence quotient (IQ) $(\beta=-0.12, P<0.001)$, item-color recall $(\beta=-0.08, P=0.010)$, and forward digit span $(\beta=-0.06, P=0.04)$. Among newly diagnosed patients, moderate/severe DKA was associated with lower item-color recall $(\beta=-0.08, P=$ 0.04). Among previously diagnosed patients, repeated DKA exposure and higher $\mathrm{HbA}_{1 \mathrm{c}}$ were independently associated with lower IQ $(\beta=-0.10$ and $\beta=-0.09$, respectively, $P<0.01$ ) and higher $\mathrm{HbA}_{1 \mathrm{c}}$ was associated with lower item-color recall $(\beta=-0.10, P=0.007)$ after hypoglycemia, diabetes duration, and socioeconomic status were accounted for.

\section{CONCLUSIONS}

A single DKA episode is associated with subtle memory declines soon after type 1 diabetes diagnosis. Sizable IQ declines are detectable in children with known diabetes, suggesting that DKA effects may be exacerbated in children with chronic exposure to hyperglycemia.

Diabetic ketoacidosis (DKA) is a common complication of type 1 diabetes (1). Of children with new onset of type 1 diabetes, $25-40 \%$ present with DKA (2). In established patients, DKA may be the consequence of poor adherence to insulin regimens, illness, or malfunction of diabetes care equipment (e.g., insulin pumps) (2). Brain injury has long been recognized as an uncommon, but serious, complication of
${ }^{1}$ Department of Psychology, University of California, Davis, Davis, CA

${ }^{2}$ Center for Mind and Brain, University of California, Davis, Davis, CA

${ }^{3}$ Department of Emergency Medicine, UC Davis Health, UC Davis School of Medicine, Sacramento, $C A$

${ }^{4}$ Department of Pediatrics, UC Davis Health, UC Davis School of Medicine, Sacramento, CA

${ }^{5}$ Division of Emergency Medicine, Department of Pediatrics, Children's Hospital Colorado, University of Colorado School of Medicine, University of Colorado Denver, Aurora, CO

${ }^{6}$ Division of Emergency Medicine, Department of Pediatrics, Children's Hospital of Philadelphia, Perelman School of Medicine, University of Pennsylvania, Philadelphia, PA

${ }^{7}$ Department of Pediatrics, University of Utah School of Medicine, Salt Lake City, UT

${ }^{8}$ Division of Emergency Medicine, Department of Pediatrics, Nationwide Children's Hospital, The Ohio State University College of Medicine, Columbus, $\mathrm{OH}$

${ }^{9}$ Departments of Emergency Medicine and Pediatrics, Rhode Island Hospital, The Warren Alpert Medical School, Brown University, Providence, RI 
DKA $(3,4)$. Recent studies, however, suggest that subtle brain injury occurs even among children with no obvious neurological symptoms during DKA $(5,6)$. Reports document that DKA is associated with alterations in memory, attention, verbal intelligence quotient (IQ) (7-12), and changes in brain microstructure (10-12).

Despite this evidence, several questions remain. First, it is unclear whether a single DKA episode results in lasting cognitive declines that are detectable shortly after the DKA episode in children with new onset of type 1 diabetes. Most studies reporting lower cognitive functioning after a single DKA episode were retrospective, included children previously diagnosed with type 1 diabetes, or documented deficits in comparison with control subjects with no diagnosis of type 1 diabetes (7-9). One small prospective study of newly diagnosed patients found that those who experienced DKA (compared with a sample of children with type 1 diabetes and no histories of DKA) showed subtle mental status and memory deficits between $48 \mathrm{~h}$ and 5 days after DKA (12). These group differences no longer persisted, however, 1 month and 6 months after diagnosis (12). Among children who experienced DKA, the severity of the DKA episode and alterations in brain microstructure detected during DKA were associated with worse cognitive functioning 6 months after diagnosis (12). It is possible that variations in DKA severity predict cognitive functioning only among children who experienced DKA, without resulting in overall differences between children with and without DKA histories. However, these findings suggest that largescale studies are necessary to fully assess the neurocognitive effects of DKA in children with newly diagnosed type 1 diabetes.

Second, reports of DKA-related cognitive deficits in children previously diagnosed with type 1 diabetes raise the question of whether these deficits may be more extensive or become more robust over time if DKA occurs in the setting of long-standing hyperglycemia (9). In a longitudinal study of children previously diagnosed with type 1 diabetes (36\% with one DKA episode), DKA exposure was associated with lower IQ 18 months later (13). However, these studies have not been able to fully account for important correlates of DKA occurrence and severity, including glycemic variability (e.g., hyperglycemia, hypoglycemia) (9), which are also associated with cognitive outcomes, and socioeconomic status (SES), which is generally associated with access to care and cognitive outcomes (14).

In the current study, we examined whether the severity of a single DKA episode was associated with cognitive deficits in children newly diagnosed with type 1 diabetes. In addition, we examined whether DKA severity of a single episode and/or repeated episodes of DKA are associated with more general cognitive deficits in children with previously diagnosed type 1 diabetes, after accounting for measures of glycemic control, such as $\mathrm{HbA}_{1 \mathrm{c}}$ and severe hypoglycemic events, duration of type 1 diabetes, and SES.

\section{RESEARCH DESIGN AND METHODS}

Children with type 1 diabetes between the ages of 6 and 18 years were recruited from sites participating in the Pediatric Emergency Care Applied Research Network (PECARN) Fluid Therapies Under Investigation in DKA (FLUID) randomized controlled trial $(15,16)$. The study was approved by the
Institutional Review Board at each site, and written informed consent was obtained from each patient's parents or guardians.

The PECARN FLUID trial compared four fluid rehydration protocols capturing variations in protocols currently used in the U.S. to treat DKA in children (15). DKA was defined by 1) blood glucose concentration $>300 \mathrm{mg} / \mathrm{dL}$ and 2 ) venous $\mathrm{pH}<7.25$ or serum bicarbonate concentration $<15$ $\mathrm{mmol} / \mathrm{L}$. Exclusion criteria have previously been described (16) and included conditions unrelated to DKA that affect mental status or cognitive abilities and/or substantial treatment for DKA prior to transfer to the study centers. All patients' medical records were reviewed by the child's primary endocrinologist to determine whether there were previous episodes of DKA or severe hypoglycemia. Parents and guardians were also queried about any hospital admissions occurring at hospitals other than the study site. If any such episodes were recalled, the child's endocrinologist reviewed these episodes with the family to verify that the admission was for DKA. For the present report, children were excluded if they experienced clinically apparent DKArelated brain injury (15) or could not return within 6 months for cognitive testing. Finally, children younger than 6 years of age were also excluded because the cognitive testing procedures used in the current study were not appropriate for younger children.

Children with type 1 diabetes, but no histories of DKA, were recruited from the pediatric diabetes clinics at the participating PECARN centers. Absence of DKA at diagnosis and between diagnosis and follow-up, and episodes of severe hypoglycemia, were verified through medical record review and confirmed by the patient's endocrinologist and family.

\footnotetext{
${ }^{10}$ Division of Emergency Medicine, Department of Pediatrics, St. Louis Children's Hospital, Washington University School of Medicine in St. Louis, St. Louis, MO

${ }^{11}$ Division of Emergency Medicine, Department of Pediatrics, Children's National Medical Center, The School of Medicine \& Health Sciences, The George Washington University, Washington, DC ${ }^{12}$ Division of Emergency Medicine, Department of Pediatrics, Ann and Robert H. Lurie Children's Hospital of Chicago, Northwestern University Feinberg School of Medicine, Chicago, IL

${ }^{13}$ Division of Emergency Medicine, Nemours/Alfred I. duPont Hospital for Children, Sidney Kimmel Medical College, Thomas Jefferson University, Philadelphia, PA

${ }^{14}$ Division of Emergency Medicine, Department of Pediatrics, Texas Children's Hospital, Baylor College of Medicine, Houston, TX

${ }^{15}$ Division of Emergency Medicine, Department of Pediatrics, Boston Children's Hospital, Harvard Medical School, Boston, MA

${ }^{16}$ Division of Emergency Medicine, Department of Pediatrics, New York Presbyterian Morgan Stanley Children's Hospital, Vagelos College of Physicians and Surgeons, Columbia University, New York, NY

${ }^{17}$ Department of Psychology, Tufts University, Medford, MA

Corresponding author: Simona Ghetti, sghetti@ ucdavis.edu
}

Received 26 January 2020 and accepted 18 August 2020

This article contains supplementary material online at https://doi.org/10.2337/figshare.12824396.

${ }^{*}$ A list of members of the Pediatric Emergency Care Applied Research Network (PECARN) DKA FLUID Study Group can be found in the supplementary material online.

(c) 2020 by the American Diabetes Association. Readers may use this article as long as the work is properly cited, the use is educational and not for profit, and the work is not altered. More information is available at https://www.diabetesjournals .org/content/license. 
Recruitment was targeted to maintain approximately equal proportions of patients with new onset of type 1 diabetes and patients with known diagnoses of type 1 diabetes in the DKA and non-DKA groups.

Patients with DKA returned between 2 and 6 months after hospital discharge for neurocognitive assessment. Patients with type 1 diabetes but no DKA histories were scheduled at their earliest convenience. The assessment for both the DKA and non-DKA groups was rescheduled if children had either hypoglycemia (glucose $<70 \mathrm{mg} / \mathrm{dL}$ ) or hyperglycemia (glucose $>350 \mathrm{mg} / \mathrm{dL}$ ) with ketosis (urine dipstick with moderate or large ketones) at the time of the visit.

\section{Outcomes}

A comprehensive neurocognitive assessment included the following: 1) color and spatial memory tasks evaluating longterm memory for pictures and association with the correct color background or spatial location with which children were initially presented (range 0-1); 2) Wechsler Abbreviated Scale of Intelligence, yielding an IQ score (range 65160) (17); and 3) digit span forward and backward task (range 0-18), evaluating short-term and working memory. Higher scores correspond to better performance. We used these measures as in our previous research $(7,15,18)$ (see supplementary materials for detailed description).

\section{Statistical Analyses}

We described the characteristics of patients with new onset of type 1 diabetes and those previously diagnosed with and without histories of DKA using relative frequencies for categorical characteristics and means and SDs for continuous characteristics. We tested for differences as a function of DKA status using likelihood ratio tests and ANOVA tests for categorical and continuous characteristics, respectively. We used $\chi^{2}$ and Wilcoxon rank sum tests to determine whether participants with DKA histories who completed the neurocognitive assessments differed from those who were lost to follow-up.

To include all patients with observed outcomes in the analyses, we used chained regression equations for imputation of missing data (19). Ten imputations were used, and results were combined using standard methods (20-22) (Supplementary
Table 1). Multiple imputation was used for predictors so that all patients with outcomes were included in the statistical analyses; imputed values were not used for outcome measures. Patients with moderate/severe DKA and mild DKA and without DKA histories were compared on all of the outcome measures with use of mixed linear models. All models included random terms for the enrolling clinical center.

Initially, models were applied to all patients and fit to item-color and itemspace memory tasks, IQ, and forward and backward digit span scores separately. The focal independent variable was DKA status, which was based on venous $\mathrm{pH}$ (or serum bicarbonate concentration in patients without a measured $\mathrm{pH}$ at presentation) in patients with DKA and was a numeric scale defined as 2, moderate/ severe $(\mathrm{pH} \leq 7.19$ or bicarbonate concentration $\leq 9 \mathrm{mmol} / \mathrm{L})$; 1 , mild $(\mathrm{pH}$ between 7.20 and 7.25 or bicarbonate between 10 and $15 \mathrm{mmol} / \mathrm{L}$ ) (23); or 0 , no DKA history $(\mathrm{pH}>7.25$ or bicarbonate $>15 \mathrm{mmol} / \mathrm{L}$ for patients with new onset of type 1 diabetes and no recorded DKA episodes for patients with established type 1 diabetes). We also included sex, SES (measured by maternal parental education on a numeric scale: $0=$ high school/GED or less, $1=$ some college/vocational school, $2=$ college degree or more), diabetes history (new onset vs. previously diagnosed), and history of severe hypoglycemic episodes (one or more vs. none). Age was included in models for item-color memory, itemspace memory, and digit span scores but not IQ, which is normalized by age. Independent variables were tested for an association with use of type III $F$ tests and a significance level of 0.05 . We calculated $95 \% \mathrm{Cls}$ for standardized regression parameters.

We subsequently applied these models separately to patients with new onset and those previously diagnosed with type 1 diabetes. We report results only for outcomes showing associations with DKA status in the initial models including all patients. In models pertaining to previously diagnosed patients, we additionally included 12-month average $\mathrm{HbA}_{1 \mathrm{c}}$, diabetes duration, and whether DKA had previously been experienced as independent variables. Again, $F$ tests were used to identify significant associations, and 95\% Cls were calculated for standardized regression coefficients. Analyses were performed using SAS software (version 9.4; SAS Institute, Cary, NC).

In exploratory analyses restricted to children with new onset of type 1 diabetes, we examined the correlation between $\mathrm{pH}$ at the time of diagnosis and IQ using Spearman correlation coefficients. In other exploratory analyses restricted to previously diagnosed patients, we included 1) the interaction between DKA severity and SES in the regression models described earlier, given differences in SES across DKA status groups in these patients, and the possibility that variability in SES was confounded with or obscured DKA status effects, and 2) the interaction between DKA exposure and age at onset of type 1 diabetes to explore whether the effects of DKA were stronger in children with onset of type 1 diabetes in early childhood.

\section{RESULTS}

A description of patient enrollment can be found in a flow diagram (Supplementary Fig. 1). Between February 2011 and September 2016, 1,249 children with DKA were enrolled in the PECARN FLUID trial and were eligible for neurocognitive followup; of these, 901 (72.1\%) returned for follow-up, and 758 (84.1\%) of patients who returned were retained for the present report based on eligibility criteria. Of these children (Table 1), 430 (56.7\%) experienced moderate or severe DKA and 328 mild DKA (43.3\%); 392 (51.7\%) had new onset of diabetes, and 366 were previously diagnosed. Among previously diagnosed patients, 270 (73.8\%) had at least one DKA episode prior to participating. Children with DKA exposure who were lost to follow-up were older, more likely to be previously diagnosed, and more likely to have higher mean $\mathrm{HbA}_{1 \mathrm{c}}$ levels than those who completed the neurocognitive assessments (Supplementary Table 2). In addition, we assessed 376 children with type 1 diabetes and no DKA histories; 199 (52.9\%) of these had new onset of type 1 diabetes.

Among newly diagnosed patients, there were no significant differences in demographic variables as a function of DKA status (Table 1). Among the children with previously diagnosed type 1 diabetes, differences as a function of DKA status were found in several demographic and diabetes-related variables (Table 1). 
Table 1-Baseline characteristics of the enrolled patients by DKA status

\begin{tabular}{|c|c|c|c|c|}
\hline & $\begin{array}{c}\text { Non-DKA } \\
(N=376)\end{array}$ & $\begin{array}{l}\text { Mild DKA } \\
(N=328)\end{array}$ & $\begin{array}{c}\text { Moderate/severe DKA } \\
\qquad(N=430)\end{array}$ & $P^{*}$ \\
\hline New onset, $N(\%)$ & 199 (52.9) & $198(60.4)$ & $194(45.1)$ & $<0.001$ \\
\hline Male, $N(\%)$ & $108(54.3)$ & 94 (47.5) & $92(47.4)$ & 0.29 \\
\hline Age at testing, mean (SD) & $11.3(3.16)$ & $11.5(2.79)$ & $11.6(2.85)$ & 0.52 \\
\hline Age at onset of diabetes, mean (SD) & $10.7(3.17)$ & $10.8(2.79)$ & $10.9(2.89)$ & 0.90 \\
\hline Glucose at neurocognitive testing, mean (SD) & $164.4(68.78)$ & $163.0(73.57)$ & $167.0(77.93)$ & 0.86 \\
\hline Any hypoglycemic episodes prior to testing, $N(\%)$ & $0(0.0)$ & $19(9.6)$ & $11(5.7)$ & $* *$ \\
\hline SES, $N(\%)^{\dagger}$ & & & & 0.74 \\
\hline High school/GED or less & $48(24.1)$ & 47 (23.9) & $57(29.4)$ & \\
\hline Some college/vocational school & $54(27.0)$ & $52(26.3)$ & $50(25.8)$ & \\
\hline College degree or more & 97 (48.9) & 98 (49.7) & $87(44.8)$ & \\
\hline Previously diagnosed, $N$ (\%) & $177(47.1)$ & $130(39.6)$ & $236(54.9)$ & \\
\hline Male, $N(\%)$ & $87(49.2)$ & $58(44.6)$ & $106(44.9)$ & 0.64 \\
\hline Age at testing, mean (SD) & $12.2(3.18)$ & $13.1(2.83)$ & $14.0(2.56)$ & $<0.001$ \\
\hline Age at onset of diabetes, mean (SD) & $8.3(3.46)$ & $6.7(3.55)$ & $7.8(3.66)$ & $<0.001$ \\
\hline Duration of diabetes in years, mean (SD) & $3.4(3.33)$ & $5.9(3.43)$ & $5.7(3.33)$ & $<0.001$ \\
\hline Glucose at neurocognitive testing, mean (SD) & $206.4(87.30)$ & $247.7(103.07)$ & $230.9(97.37)$ & $<0.001$ \\
\hline Any hypoglycemic episodes prior to testing, $N(\%)$ & $12(6.9)$ & $35(27.1)$ & $48(20.4)$ & $<0.001$ \\
\hline One or more additional DKA episodes, $N(\%)$ & $0(0.0)$ & $101(77.5)$ & $169(71.5)$ & $* *$ \\
\hline 12-month average $\mathrm{HbA}_{1 \mathrm{c}}$ result, mean (SD) & $8.3(1.34)$ & $10.2(1.91)$ & $10.6(1.75)$ & $<0.001$ \\
\hline SES, $N(\%)^{\dagger}$ & & & & 0.003 \\
\hline High school/GED or less & $32(18.0)$ & $47(36.4)$ & $100(42.5)$ & \\
\hline Some college/vocational school & $48(27.2)$ & $46(35.6)$ & $75(31.6)$ & \\
\hline College degree or more & $97(54.8)$ & $36(28.0)$ & $61(25.9)$ & \\
\hline
\end{tabular}

$* P$ values are from likelihood ratio $\chi^{2}$ tests for categorical characteristics [those with $N(\%)$ ] and ANOVA $F$ tests for continuous characteristics [those with mean (SD)], using methods for combining results across multiple imputed data sets. ${ }^{* *} P$ values were not calculated due to design-imposed relationships with DKA status. †Maternal education if documented. If not, imputed using paternal education and household income in a multinomial regression model.

The first analysis included all participants (i.e., both newly diagnosed and previously diagnosed patients) and compared cognitive outcomes in children with moderate or severe DKA, mild DKA, or no DKA history. Additional variables in the model included age at testing, sex, SES, experience of severe hypoglycemia, and whether children were newly or previously diagnosed. DKA status was associated with lower scores in overall $\mathrm{IQ}$, memory for item-color associations, and forward digit span recall (Table 2). Backward digit span recall and memory for item-space relations did not differ between moderate/severe DKA, mild DKA, and non-DKA groups (Supplementary Table 3). Children with previously diagnosed type 1 diabetes showed lower performance compared with children with new onset in the item-color associations and overall IQ (Table 2).

Table 2-Regression models applied to all patients: standardized regression coefficients and $95 \%$ CIs*† and adjusted means* + and raw means by DKA status

\begin{tabular}{|c|c|c|c|c|c|c|c|c|}
\hline & \multicolumn{3}{|c|}{ Color task } & \multicolumn{3}{|c|}{ IQ } & \multicolumn{2}{|c|}{ Digit span recall: forward } \\
\hline & \multicolumn{2}{|c|}{ Coefficient $(95 \% \mathrm{Cl})$} & $P$ & \multicolumn{2}{|c|}{ Coefficient $(95 \% \mathrm{Cl})$} & $P$ & Coefficient $(95 \% \mathrm{Cl})$ & $P$ \\
\hline DKA status $¥$ & \multicolumn{2}{|c|}{$-0.08(-0.13,-0.02)$} & 0.010 & \multicolumn{2}{|c|}{$-0.12(-0.19,-0.06)$} & $<0.001$ & $-0.06(-0.12,-0.00)$ & 0.04 \\
\hline Male & \multicolumn{2}{|c|}{$-0.05(-0.10,0.00)$} & 0.07 & \multicolumn{2}{|c|}{$0.01(-0.04,0.07)$} & 0.67 & $-0.00(-0.06,0.05)$ & 0.88 \\
\hline Age & \multicolumn{2}{|c|}{$0.47(0.41,0.53)$} & $<0.001$ & \multicolumn{2}{|c|}{ Not estimated } & & $0.41(0.35,0.47)$ & $<0.001$ \\
\hline Previously diagnosed\# & \multicolumn{2}{|c|}{$-0.07(-0.13,-0.01)$} & 0.02 & \multicolumn{2}{|c|}{$-0.08(-0.14,-0.03)$} & 0.004 & $-0.05(-0.11,0.01)$ & 0.10 \\
\hline SES§ & \multicolumn{2}{|c|}{$0.10(0.04,0.16)$} & $<0.001$ & \multicolumn{2}{|c|}{$0.32(0.26,0.38)$} & $<0.001$ & $0.12(0.06,0.17)$ & $<0.001$ \\
\hline \multirow[t]{2}{*}{ Previous severe hypoglycemia } & \multicolumn{2}{|c|}{$0.01(-0.05,0.07)$} & 0.72 & \multicolumn{2}{|c|}{$-0.03(-0.09,0.03)$} & 0.30 & $0.00(-0.05,0.06)$ & 0.96 \\
\hline & \multicolumn{3}{|c|}{ Color task } & \multicolumn{3}{|l|}{ IQ } & \multicolumn{2}{|c|}{ Digit span recall: forward } \\
\hline DKA status & Adjusted mean (SE) & \multicolumn{2}{|c|}{ Mean (SD) } & Adjusted mean (SE) & \multicolumn{2}{|c|}{ Mean (SD) } & Adjusted mean (SE) & Mean (SD) \\
\hline No DKA & $0.50(0.012)$ & \multicolumn{2}{|c|}{$0.50(0.180)$} & $104.6(1.15)$ & \multicolumn{2}{|c|}{$107.3(13.95)$} & $8.5(0.14)$ & $8.6(2.25)$ \\
\hline Mild DKA & $0.48(0.010)$ & \multicolumn{2}{|c|}{$0.49(0.170)$} & $102.6(1.02)$ & \multicolumn{2}{|c|}{$103.7(13.46)$} & $8.4(0.12)$ & $8.2(2.11)$ \\
\hline Moderate/severe DKA & $0.47(0.011)$ & \multicolumn{2}{|c|}{$0.49(0.168)$} & $100.7(1.12)$ & \multicolumn{2}{|c|}{$101.2(12.31)$} & $8.2(0.13)$ & $8.5(2.06)$ \\
\hline
\end{tabular}

*Regression coefficients are standardized and represent the mean change in outcome associated with a 1-SD change in a continuous factor or the change associated with that level (male, previously diagnosed, previous severe hypoglycemia) for binary variables. Note that the estimated change for the reference value (female, new onset, no hypoglycemia) is -1 times the coefficient. Adjusted means average over sex, previous diagnosis, and previous severe hypoglycemia and assume age $=12$ and $S E S=1$. †Random effect of site included in each model. $¥ D$ DKA status is on a numeric scale: $0=$ no DKA, $1=$ mild DKA, 2 = moderate/severe DKA. \#Previously diagnosed: $0=$ newly diagnosed patients and $1=$ previously diagnosed patients, allowing for the comparison of these two groups. §SES is indicated by maternal education and is on a numeric scale: $0=$ high school/GED or less, $1=$ some college/ vocational school, 2 = college degree or more; when data were missing, income and paternal education were used to impute SES. 
Table 3-Regression models applied separately to patients with new-onset and previously diagnosed diabetes

\begin{tabular}{|c|c|c|c|c|c|c|c|c|c|}
\hline \multirow{2}{*}{ New-onset patients } & \multicolumn{4}{|c|}{ Color task } & \multicolumn{3}{|c|}{ IQ } & \multicolumn{2}{|c|}{ Digit span recall: forward } \\
\hline & \multicolumn{2}{|c|}{ Coefficient $(95 \% \mathrm{Cl})$} & \multicolumn{2}{|c|}{$P$} & \multicolumn{2}{|c|}{ Coefficient $(95 \% \mathrm{Cl})$} & $P$ & Coefficient $(95 \% \mathrm{Cl})$ & $P$ \\
\hline DKA status $¥$ & \multicolumn{2}{|c|}{$-0.08(-0.16,-0.00)$} & \multicolumn{2}{|c|}{0.04} & \multicolumn{2}{|c|}{$-0.06(-0.14,0.02)$} & 0.13 & $-0.03(-0.11,0.05)$ & 0.44 \\
\hline Male & \multicolumn{2}{|c|}{$-0.06(-0.13,0.02)$} & \multicolumn{2}{|c|}{0.14} & \multicolumn{2}{|c|}{$0.02(-0.06,0.09)$} & 0.63 & $0.03(-0.04,0.11)$ & 0.37 \\
\hline Age & \multicolumn{2}{|c|}{$0.45(0.37,0.53)$} & \multicolumn{2}{|c|}{$<0.001$} & \multicolumn{2}{|c|}{ Not estimated } & & $0.39(0.31,0.47)$ & $<0.001$ \\
\hline SES§ & \multicolumn{2}{|c|}{$0.11(0.04,0.19)$} & \multicolumn{2}{|c|}{0.003} & \multicolumn{2}{|c|}{$0.34(0.26,0.42)$} & $<0.001$ & $0.12(0.04,0.19)$ & 0.003 \\
\hline \multirow[t]{2}{*}{ Previous severe hypoglycemi } & \multicolumn{2}{|c|}{$0.03(-0.08,0.14)$} & \multicolumn{2}{|c|}{0.61} & \multicolumn{2}{|c|}{$-0.07(-0.18,0.04)$} & 0.21 & $-0.06(-0.17,0.05)$ & 0.28 \\
\hline & \multicolumn{4}{|c|}{ Color task } & \multicolumn{3}{|l|}{ IQ } & \multicolumn{2}{|c|}{ Digit span recall: forward } \\
\hline DKA status & Adjusted mean (SE) & \multicolumn{2}{|c|}{ Mean (SD) } & \multicolumn{2}{|c|}{ Adjusted mean (SE) } & \multicolumn{2}{|c|}{ Mean (SD) } & Adjusted mean (SE) & Mean (SD) \\
\hline No DKA & $0.52(0.019)$ & $0.49(0$. & 175) & & $103.9(1.63)$ & 106.5 & (14.18) & $8.3(0.23)$ & $8.5(2.25)$ \\
\hline Mild DKA & $0.50(0.016)$ & $0.50(0$. & 159) & & $102.9(1.43)$ & 106.5 & (13.15) & $8.3(0.20)$ & $8.2(2.06)$ \\
\hline Moderate/severe DKA & $0.48(0.017)$ & $0.47(0$. & 171) & & $101.9(1.53)$ & 103.5 & (12.18) & $8.2(0.21)$ & $8.4(2.09)$ \\
\hline & & or task & & & & Q & & Digit span recall: & : forward \\
\hline Previously diagnosed patients & Coefficient ( & $5 \% \mathrm{Cl})$ & & & Coefficient (95 & $6 \mathrm{Cl})$ & $P$ & Coefficient $(95 \% \mathrm{Cl})$ & $P$ \\
\hline DKA status $¥$ & $-0.02(-0.1$ & $0.08)$ & 0. & & $-0.08(-0.18$ & $0.02)$ & 0.14 & $-0.08(-0.18,0.01)$ & 0.10 \\
\hline Male & $-0.04(-0.1$ & , 0.04) & 0. & & $-0.00(-0.08$ & $0.07)$ & 0.94 & $-0.04(-0.12,0.04)$ & 0.30 \\
\hline Age & $0.52(0.42$ & $0.62)$ & $<0$ & 001 & Not estimat & & & $0.41(0.32,0.51)$ & $<0.001$ \\
\hline SES§ & $0.06(-0.02$ & $0.15)$ & 0. & & $0.27(0.18,0$ & & $<0.001$ & $0.12(0.04,0.21)$ & 0.005 \\
\hline Previous severe hypoglycemi & $0.01(-0.06$ & $0.09)$ & 0. & & $0.03(-0.04$ & $.10)$ & 0.45 & $0.03(-0.04,0.10)$ & 0.47 \\
\hline Mean $\mathrm{HbA}_{1 \mathrm{c}}$ & $-0.10(-0.1)$ & $-0.03)$ & 0.0 & & $-0.09(-0.16$ & $-0.02)$ & 0.008 & $0.03(-0.04,0.09)$ & 0.45 \\
\hline Diabetes duration & $-0.00(-0.0$ & , 0.09) & $>0$ & & $-0.03(-0.12$ & $0.05)$ & 0.42 & $0.02(-0.07,0.11)$ & 0.62 \\
\hline Previous DKA episodes & $-0.01(-0.0$ & $0.07)$ & 0. & & $-0.10(-0.18$ & $-0.03)$ & 0.009 & $-0.04(-0.12,0.04)$ & 0.35 \\
\hline & Color tas & & & & IQ & & & Digit span recall: $f$ & forward \\
\hline DKA status & Adjusted mean (SE) & Mean ( & & & usted mean (SE) & $\mathrm{Me}$ & $(S D)$ & Adjusted mean (SE) & Mean (SD) \\
\hline No DKA & $0.47(0.018)$ & $0.51(0$. & 185) & & $103.0(1.46)$ & 107.6 & (13.71) & $8.5(0.20)$ & $8.7(2.25)$ \\
\hline Mild DKA & $0.46(0.013)$ & $0.47(0$. & 185) & & 101.8 (1.14) & 99.2 & (12.78) & $8.3(0.14)$ & $8.3(2.18)$ \\
\hline Moderate/severe DKA & $0.46(0.015)$ & $0.51(0$ & 164) & & $100.6(1.34)$ & 98.9 & 11.99) & $8.0(0.17)$ & $8.6(2.03)$ \\
\hline
\end{tabular}

Data shown are regression coefficients and $95 \% \mathrm{Cls}$ and adjusted means and raw means as a function of DKA status (random effect of site included in each model). Regression coefficients are standardized and represent the mean change in outcome associated with a 1-SD change in a continuous factor or the change associated with that level (male, previous severe hypoglycemia) for binary variables. Note that the estimated change for the reference value (female and no hypoglycemia) is -1 times the coefficient. Adjusted means are conditional on applicable covariates as follows: average sex and previous severe hypoglycemia, age $=12, \mathrm{SES}=1$, mean $\mathrm{HbA}_{1 \mathrm{c}}=9.6 \%$, diabetes duration $=5$ years, and one to two previous DKA episodes. $¥ \mathrm{DKA}$ status is on a numeric scale: $0=$ no DKA, $1=$ mild DKA, $2=$ moderate/severe DKA. $§ S E S$ is indicated by maternal education and is on a numeric scale: $0=$ high school/GED or less, $1=$ some college/vocational school, 2 = college degree or more; when data were missing, income and paternal education were used to impute SES.

The next set of analyses examined performance separately for patients with new onset of type 1 diabetes and those previously diagnosed. We limited these analyses to the outcomes that showed an overall effect of DKA status in the previous analysis, namely, item-color memory, IQ, and forward digit span.

\section{Patients With Newly Diagnosed Type 1 Diabetes}

The regression models including newly diagnosed patients revealed significant differences as a function of DKA status at presentation for item-color association memory but not for IQ and forward digit span (Table 3). In exploratory analyses restricted to patients who presented with DKA, lower venous $\mathrm{pH}$ was associated with lower IQ $(r=0.18, P=0.001)$.
This correlation retained its significance when we controlled for sex, SES, and glucose level at diagnosis of DKA and at testing $(r=0.16, P=0.003)$.

\section{Patients With Previously Diagnosed Type 1 Diabetes}

The models examining previously diagnosed patients revealed that DKA status was not related to any of the cognitive outcomes but that greater number of previous DKA episodes and higher $\mathrm{HbA}_{1 \mathrm{c}}$ were independently and negatively related to IQ (Table 3). $\mathrm{HbA}_{1 \mathrm{c}}$ was also negatively related to item-color memory (Table 3). However, DKA status was associated with both mean $\mathrm{HbA}_{1 \mathrm{c}}$ and SES among previously diagnosed patients in bivariable analyses (Table 1), limiting our ability to isolate the unique effects of
DKA among patients with poor glycemic control. Furthermore, the associations between DKA status and SES among previously diagnosed patients (Table 1 ) and SES and neurocognitive outcomes (Tables 2 and 3) raise the question of whether preexisting differences among patient groups may account for or obscure differences as a function of DKA status. To address this question, we conducted exploratory analyses in which an interaction term involving DKA status and SES was added to the regression model for previously diagnosed patients. These analyses revealed a significant interaction effect (Supplementary Table 4), such that DKA status was associated with lower IQ among children with higher SES (Fig. 1), whereas patients with lower SES exhibited lower IQ regardless of DKA 


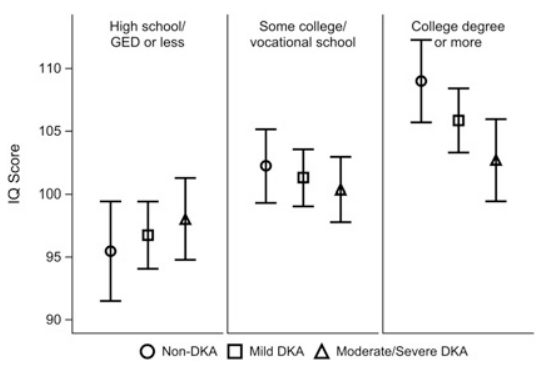

Figure 1-Interaction effect between DKA status and levels of SES among previously diagnosed patients. Estimates and $95 \% \mathrm{Cls}$ of IQ average over other effects in a multivariable mixed linear regression model, which included sex, duration of diabetes, previous DKA episodes, history of severe hypoglycemia, $\mathrm{HbA}_{1 \mathrm{c}}$, and study site. GED, General Educational Development.

status. Finally, the broad range of age of onset of type 1 diabetes in this group and evidence that early age of onset may result in worse cognitive outcomes (7) motivated the examination of interaction effects between age of onset and DKA exposure. We found an interaction effect (Supplementary Table 5) such that DKA exposure was associated with stronger decline in item-color memory if onset occurred prior to the child's fourth birthday.

\section{CONCLUSIONS}

Since our initial report of memory declines following one single DKA episode (7), several studies have suggested that DKA is an important predictor of cognitive decline in children with type 1 diabetes, even in the absence of overt DKA-related brain injury (8-12). These studies, however, were retrospective, included small samples, or did not fully account for variability in glycemic control and other important variables, including SES (8-12). To our knowledge, this is the first large-scale, prospective study of DKA to differentiate associations between DKA status and cognition in children with new onset and children with a previous diagnosis of type 1 diabetes. When both groups of patients were combined, we found lower performance in measures of short-term memory, long-term memory, and overall IQ among children who experienced moderate/severe DKA. Analyses separating new onset from previously diagnosed patients provided a more nuanced picture.

Among newly diagnosed patients, moderate/severe DKA was associated with lower item-color memory. Greater acidosis was also associated with lower $\mathrm{IQ}$ in an analysis restricted to newly diagnosed patients who experienced DKA. In addition, among newly diagnosed patients, there were no differences in SES or in any other examined variable by DKA status (moderate/severe DKA, mild DKA, no DKA), suggesting that DKA-related effects are not due to preexisting differences among these groups of newly diagnosed patients. Instead, these findings suggest that DKA-related deficits are detectable soon after a single moderate/ severe DKA episode.

Previous literature does not paint a coherent picture about whether a single DKA episode at the time of diagnosis of type 1 diabetes suffices to generate cognitive declines that are detectable soon after recovery. Most studies reporting the effects of a single DKA episode were retrospective or included patients with known type 1 diabetes (7-9), making it impossible to address this question directly. In a relatively small sample of prospectively examined newly diagnosed patients, those who presented with DKA exhibited cognitive declines within days of the DKA episode; however, these group differences did not persist after 1-6 months (12). Interestingly, DKA severity and alterations in brain white matter integrity observed during DKA were associated with lower cognitive performance after 6 months. Consistent with these results, we found that in children with new onset of type 1 diabetes, moderate/severe DKA was associated with lower item-color memory, and lower $\mathrm{pH}$ in DKA patients was correlated with lower IQ, 2-6 months after diagnosis. Future research using a more extensive IQ measure may provide a more precise characterization of cognitive declines in individual subtests of IQ.

In contrast to our findings, a recent population study of Danish children did not find overall differences between children with and without DKA exposure in performance on standardized achievement tests (24). However, DKA was documented in only $18 \%$ of the patients, resulting in a sample that was substantially smaller than that examined here. Furthermore, the severity of DKA was not documented in this population study. If DKA was largely prevented or treated while still mild, neurocognitive deficits may have been less likely to emerge in that sample.
Among children previously diagnosed with type 1 diabetes, our findings show that elevated $\mathrm{HbA}_{1 \mathrm{c}}$ was associated with lower item-color memory; overall DKA exposure was associated with lower item-color memory only among those who were diagnosed prior to age 4 years, consistent with other reports of increased vulnerability following early diagnosis $(7,25)$. Previous DKA exposure and elevated $\mathrm{HbA}_{1 \mathrm{c}}$ were independently associated with lower IQ. DKA severity in the episode targeted in our study was associated with lower IQ when these other factors were accounted for only among children with higher SES; lower-SES patients showed lower performance overall, which was not further impacted by DKA severity. This finding counters the hypothesis that the effects of DKA status entirely reflect preexisting group differences including a greater probability of experiencing DKA (or severe DKA) in lowSES children, who are also more likely to exhibit lower cognitive performance. Our results suggest instead that the unique effects of DKA severity of a recent episode may be obscured or subsumed by other associated variables or risk factors among previously diagnosed patients.

Other evidence $(13,26)$ suggests that DKA severity in previously diagnosed patients is associated with lower IQ 18 months later. In that research, however, glycemic variability and SES were not fully accounted for, preventing firm conclusions about the unique effects of DKA. The IQ declines associated with DKA over an 18-month period $(13,26)$ highlight that these deficits may worsen over time, placing children at increased risk for academic difficulties or problems managing type 1 diabetes independently.

The examination of a large cohort allowed us to capture more complex associations among relevant variables. The strong associations of DKA severity, SES, and poor glycemic control underscore that measures of cumulative DKA exposure and glycemic control may capture much of the variance in behavioral performance and that patients with repeated DKA exposure and poorly controlled type 1 diabetes are at substantial risk of cognitive deficits.

Our findings confirm that subtle brain injuries may occur as a result of DKA, but the underlying mechanism is still unclear. Previous hypotheses suggested that osmotic declines during DKA treatment 
might cause cerebral edema resulting in cerebral injury. These hypotheses were largely disproven in our recent randomized controlled trial, which found that variations in intravenous fluid treatment regimens for DKA do not substantially affect neurocognitive outcomes in children (15). Thus, other mechanisms should be considered. Severe DKA is associated with alterations in cerebral perfusion and systemic increases in levels of inflammatory mediators (27-31). In addition, studies in a juvenile rat DKA model demonstrate that a single DKA episode causes a neuroinflammatory response that involves reactive astrogliosis, activation of microglia in the hippocampus, and long-term cortical neuronal loss (31). These findings suggest that inflammatory processes triggered by DKA might cause ongoing neuroinflammation that leads to long-term cognitive decline. Future studies are necessary to determine whether events similar to those documented in the rat model are responsible for cognitive deficits in children with DKA exposure. Observed deficits involve memory and IQ, which have been associated with hippocampal integrity (32). Thus, examination of the links between markers of neuroinflammation, structural changes in the hippocampus, and behavioral outcomes may be particularly promising.

The current study has some limitations. First, as in most studies of children with type 1 diabetes, we have no measures of cognitive functioning prior to diagnosis, raising the issue of whether preexisting cognitive differences between groups could account for some of our observations. However, associations of cognitive outcomes with DKA severity among patients with new onset of diabetes, and the different pattern of results observed in these patients compared with those with a previous diagnosis of type 1 diabetes, attenuate this concern. Newly diagnosed and previously diagnosed children were largely comparable in age, sex, and SES and were recruited at the same centers using the same exclusion criteria (e.g., exclusion of children with known learning disabilities). Nevertheless, a clearer understanding of the effects of DKA might be achieved in future longitudinal studies if neurocognitive assessments begin at diagnosis and DKA episodes occur later in the course of disease. Second, children previously diagnosed with type 1 diabetes who had histories of DKA and poor glycemic control were more likely to be lost to follow-up than newly diagnosed patients and those with good glycemic control. Thus, our reported effects may underestimate cognitive alterations in the overall population because those children lost to follow-up may be at higher risk for cognitive declines. Comparable DKA severity between patients who completed the study and those lost to follow-up, however, suggests that the reported effects of DKA status are generalizable. Third, we relied on medical record reviews and, in part, on parents' or guardians' recollections of previous DKA episodes and severe hypoglycemic events to classify patients according to these variables. It is possible that some additional episodes of DKA or severe hypoglycemia may have been missed among previously diagnosed patients. The relatively uncommon occurrence of severe hypoglycemia in this study and its association with other variables may have limited our ability to detect associations between severe hypoglycemia and alterations in cognition. Fourth, among patients with new onset of type 1 diabetes, $\mathrm{HbA}_{1 \mathrm{c}}$ levels were not routinely measured at diagnosis. Although $\mathrm{HbA}_{1 \mathrm{c}}$ levels at diagnosis are not predictive of future glycemic control, recent studies suggest that glycemic exposure prior to diagnosis may impact cognitive outcomes (8), and we were unable to account for this effect. Finally, our exclusion of children with learning disabilities prevents us from drawing conclusions about the effects of DKA in patients who may be even more vulnerable to alteration of cognitive development than typically developing children. Conversely, lack of inclusion of a control group of children without diabetes prevents us from drawing conclusions about general consequences of type 1 diabetes.

Overall, our results suggest that DKA is associated with cognitive alterations in children with type 1 diabetes, including those with newly diagnosed type 1 diabetes. The neurocognitive effects of DKA in children with preexisting type 1 diabetes should be evaluated in the context of additional variables, including repeated DKA exposure and glycemic control. Our results emphasize the importance of prevention of DKA in children with known type 1 diabetes and of prompt diagnosis during new onset of type 1 diabetes before the development of DKA.

Acknowledgments. The authors thank Marci Fjelstad and Amy Watson from the PECARN Data Coordinating Center for their assistance; the research coordinators in PECARN, without whom this trial would not have been possible; the clinicians in PECARN who enrolled children into this study; the members of the data and safety monitoring board (Roger Lewis [Department of Emergency Medicine, Harbor-UCLA Medical Center], Jeffrey Blumer [School of Medicine, Case Western Reserve University], Andrew Bremer [National Institute of Child and Human Development], Thomas Cook [Department of Biostatistics and Medical Informatics, University of Wisconsin], and Beth Slomine [Department of Psychiatry, Johns Hopkins University School of Medicine]); and the members of the study outcome adjudication committee (Kathleen Meert [Department of Pediatrics, Children's Hospital of Michigan], Jerry Zimmerman [Center for Clinical and Translational Research, Seattle Children's Hospital], and Robert Hickey [Division of Pediatric Emergency Medicine, Children's Hospital of Pittsburgh]).

Funding. This study was supported by a grant from the Eunice Kennedy Shriver National Institute of Child Health and Human Development (U01HD062417). This project was also supported in part by the Health Resources and Services Administration, Maternal and Child Health Bureau, and Emergency Medical Services for Children Network Development Demonstration Program under cooperative agreement numbers U03MC00008, U03MC00001, U03MC00003, U03MC00006, U03MC00007, U03MC22684, and U03MC22685.

This information or content and conclusions are those of the authors and should not be construed as the official position or policy of, nor should any endorsements be inferred by, Health Resources and Services Administration, Health and Human Services, or the U.S. government.

Duality of Interest. No potential conflicts of interest relevant to this article were reported. Author Contributions. S.G. conceived and designed the study, supervised training of study personnel and neurocognitive data collection, contributed to data analysis, drafted the initial manuscript, and approved the final manuscript. N.K. conceived and designed the study, obtained grant funding, supervised training of study personnel, supervised patient enrollment and data abstraction, contributed to data analysis, contributed to the initial draft, and revised and approved the final manuscript. A.R. supervised patient enrollment and data abstraction, contributed to study design, and revised and approved the final manuscript. S.R.M. supervised patient enrollment and data abstraction, contributed to the study design, and revised and approved the final manuscript. J.E.S. supervised patient enrollment and data abstraction, contributed to study design, and revised and approved the final manuscript. M.J.S. supervised patient enrollment and data abstraction, contributed to study design, and revised and 
approved the final manuscript. A.G. supervised patient enrollment and data abstraction, contributed to study design, and revised and approved the final manuscript. K.S.Q. supervised patient enrollment and data abstraction, contributed to study design, and revised and approved the final manuscript. K.M.B. supervised patient enrollment and data abstraction, contributed to study design, and revised and approved the final manuscript. J.L.T. supervised patient enrollment and data abstraction, contributed to study design, and revised and approved the final manuscript. L.T. supervised patient enrollment and data abstraction, contributed to study design, and revised and approved the final manuscript. A.D.D. supervised patient enrollment and data abstraction, contributed to study design, and revised and approved the final manuscript. J.K.M. supervised patient enrollment and data abstraction, contributed to study design, and revised and approved the final manuscript. L.E.N. supervised patient enrollment and data abstraction, contributed to study design, and revised and approved the final manuscript. M.Y.K. supervised patient enrollment and data abstraction, contributed to study design, and revised and approved the final manuscript. C.S.P. trained study personnel, assisted in neurocognitive data collection and processing, and reviewed and approved the final manuscript. C.S.O. conducted data analyses and drafted tables and figures for the final manuscript and revised and approved the final manuscript. T.C.C. supervised data analyses and drafting of tables and figures for the final manuscript and revised and approved the final manuscript. N.S.G. conceived and designed the study, obtained grant funding, supervised training of study personnel, supervised patient enrollment and data abstraction, contributed to data analysis, contributed to the initial draft, and revised and approved the final manuscript. C.S.O. and T.C.C. are the guarantors of this work and, as such, had full access to all the data in the study and take responsibility for the integrity of the data and the accuracy of the data analysis.

\section{References}

1. Sperling MA. Diabetic ketoacidosis. Pediatr Clin North Am 1984;31:591-610

2. Faich GA, Fishbein HA, Ellis SE. The epidemiology of diabetic acidosis: a population-based study. Am J Epidemiol 1983;117:551-558

3. Glaser N, Barnett P, McCaslin I, et al.; Pediatric Emergency Medicine Collaborative Research Committee of the American Academy of Pediatrics. Risk factors for cerebral edema in children with diabetic ketoacidosis. N Engl J Med 2001; 344:264-269

4. Rosenbloom AL. Intracerebral crises during treatment of diabetic ketoacidosis. Diabetes Care 1990;13:22-33
5. Wootton-Gorges SL, Buonocore MH, Kuppermann $\mathrm{N}$, et al. Cerebral proton magnetic resonance spectroscopy in children with diabetic ketoacidosis. AJNR Am J Neuroradiol 2007;28:895-899 6. Glaser NS, Wootton-Gorges SL, Buonocore $\mathrm{MH}$, et al. Frequency of sub-clinical cerebral edema in children with diabetic ketoacidosis. Pediatr Diabetes 2006;7:75-80

7. Ghetti S, Lee JK, Sims CE, Demaster DM, Glaser NS. Diabetic ketoacidosis and memory dysfunction in children with type 1 diabetes. J Pediatr 2010;156:109-114

8. Semenkovich K, Bischoff A, Doty T, et al. Clinical presentation and memory function in youth with type 1 diabetes. Pediatr Diabetes 2016:17:492-499

9. Ryan CM, van Duinkerken E, Rosano C. Neurocognitive consequences of diabetes. Am Psychol 2016;71:563-576

10. Siller AF, Lugar $H$, Rutlin J, et al. Severity of clinical presentation in youth with type $1 \mathrm{di}$ abetes is associated with differences in brain structure. Pediatr Diabetes 2017;18:686-695

11. Mackay MT, Molesworth C, Northam EA, Inder TE, Cameron FJ; DKA Brain Injury Study Group. Diabetic ketoacidosis and electroencephalographic changes in newly diagnosed pediatric patients. Pediatr Diabetes 2016;17:244-248

12. Cameron FJ, Scratch SE, Nadebaum C, et al.; DKA Brain Injury Study Group. Neurological consequences of diabetic ketoacidosis at initial presentation of type 1 diabetes in a prospective cohort study of children. Diabetes Care 2014;37: 1554-1562

13. Cato MA, Mauras N, Mazaika P, et al.; Diabetes Research in Children Network. Longitudinal evaluation of cognitive functioning in young children with type 1 diabetes over 18 months. J Int Neuropsychol Soc 2016;22:293-302

14. Bradley RH, Corwyn RF. Socioeconomic status and child development. Annu Rev Psychol 2002;53:371-399

15. Kuppermann N, Ghetti S, Schunk JE, et al Clinical trial of fluid infusion rates for pediatric diabetic ketoacidosis. N Engl J Med 2018;378: 2275-2287

16. Glaser NS, Ghetti S, Casper TC, Dean JM, Kuppermann N; Pediatric Emergency Care Applied Research Network (PECARN) DKA FLUID Study Group. Pediatric diabetic ketoacidosis, fluid therapy, and cerebral injury: the design of a factorial randomized controlled trial. Pediatr Diabetes 2013;14:435-446

17. Wechsler D. Wechsler Abbreviated Scale of Intelligence. San Antonio, TX, The Psychological Corporation, 1999

18. Perry CS III, Rapinett G, Glaser NS, Ghetti S. Hydration status moderates the effects of drinking water on children's cognitive performance. Appetite 2015;95:520-527

19. Raghunathan TE, Lepkowski JM, van Hoewyk J, Solenberger P. A multivariate technique for multiply imputing missing values using a sequence of regression Mmodels. Surv Methodol 2001;27: 85-95

20. Rubin DB. Multiple Imputation for Nonresponse in Surveys. New York, John Wiley \& Sons, 1987

21. Schafer JL. Multiple imputation: a primer. Stat Methods Med Res 1999;8:3-15

22. Von Hippel PT. Regression with Missing Y's: an improved strategy for analyzing multiply imputed data. Sociol Methodol 2007;37:83117

23. Wolfsdorf JI, Glaser N, Agus M, et al. ISPAD Clinical Practice Consensus Guidelines 2018: diabetic ketoacidosis and the hyperglycemic hyperosmolar state. Pediatr Diabetes 2018;19(Suppl. 27): 155-177

24. Skipper N, Gaulke A, Sildorf SM, Eriksen TM, Nielsen NF, Svensson J. Association of type 1 diabetes with standardized test scores of Danish schoolchildren. JAMA 2019;321: 484-492

25. Lacy ME, Gilsanz P, Eng CW, Beeri MS, Karter AJ, Whitmer RA. Recurrent diabetic ketoacidosis and cognitive function among older adults with type 1 diabetes: findings from the Study of Longevity in Diabetes. BMJ Open Diabetes Res Care 2020;8:e001173

26. Aye T, Mazaika PK, Mauras N, et al.; Diabetes Research in Children Network (DirecNet) Study Group. Impact of early diabetic ketoacidosis on the developing brain. Diabetes Care 2019;42: 443-449

27. Hoffman WH, Passmore GG, Hannon DW, et al. Increased systemic Th17 cytokines are associated with diastolic dysfunction in children and adolescents with diabetic ketoacidosis. PLoS One 2013;8:e71905

28. Omatsu T, Cepinskas G, Clarson C, et al.; Canadian Critical Care Translational Biology Group. CXCL1/CXCL8 (GRO $\alpha /$ IL-8) in human diabetic ketoacidosis plasma facilitates leukocyte recruitment to cerebrovascular endothelium in vitro. Am J Physiol Endocrinol Metab 2014; 306:E1077-E1084

29. Stentz FB, Umpierrez GE, Cuervo R, Kitabchi AE. Proinflammatory cytokines, markers of cardiovascular risks, oxidative stress, and lipid peroxidation in patients with hyperglycemic crises. Diabetes 2004;53:2079-2086

30. Garro A, Chodobski A, Szmydynger-Chodobska J, et al. Diabetic ketoacidosis results in elevation of plasma levels of matrix metalloproteinase-9 in children with type 1 diabetes. Pediatr Diabetes 2017;18:95-102

31. Lo W, O'Donnell M, Tancredi D, Orgain M, Glaser N. Diabetic ketoacidosis in juvenile rats is associated with reactive gliosis and activation of microglia in the hippocampus. Pediatr Diabetes 2016;17:127-139

32. Demaster DM, Ghetti S. Developmental differences in hippocampal and cortical contributions to episodic retrieval. Cortex 2013;49: 1482-1493 